\title{
Preliminary observations on an isolate from synovial fluid of patients with rheumatoid arthritis
}

\author{
JEAN G. MARKHAM AND D. B. MYERS \\ From the Laboratory Services Department, Dunedin Hospital and the Department of Medicine, \\ Wellcome Medical Research Institute, University of Otago, New Zealand
}

\begin{abstract}
Markham, J. G., and Myers, D. B. (1976). Annals of the Rheumatic Diseases, 35, 1-7. Preliminary observations on an isolate from synovial fluid of patients with rheumatoid arthritis. A very slow-growing micro-organism has been isolated from the synovial fluid of patients suffering from rheumatoid arthritis. This organism exhibits some of the properties of a mycoplasma such as growth in the presence of penicillin, and the requirement of serum and yeast, but generally does not behave like any known species of this group. Colonies resemble some types of $\mathrm{T}$-mycoplasma in morphology and size. Scanning electron microscopy showed that the size of the components of these colonies was exceedingly small.
\end{abstract}

For many years investigators have searched for an organism which might act as a triggering mechanism or be a contributory factor in the development of rheumatoid arthritis (RA). An earlier investigation by Highton and others (1966) reported that a specific strain of mycoplasma was grown from a case of monarticular RA, which later developed a rising rheumatoid factor titre and progressive joint disease. Recently, however, Middleton and Highton (1975) stated that in a further series of 41 patients attempts to isolate mycoplasmas from patients with RA failed when established methods of culture were used.

In this study the investigation was continued using material from patients in the acute stage of RA where possible. Synovial fluid specimens were cultured in the latest media developed for fastidious mycoplasma and the isolates examined with a scanning electron microscope giving high resolution, thus providing detail of structure, size, and shape of the components of the colonies impossible to achieve with a conventional light microscope. Biochemical studies were also done to attempt to identify the isolates.

\section{Materials and methods}

PATIENTS AND MATERIAL EXAMINED

Patients were examined at the Dunedin Public Hospital. 11 cases of RA were diagnosed and 1 ankylosing spondylitis. Synovial fluid was removed during therapy for investigation. Included in the material investigated were 2 broth cultures, 52-M and 56-M (kindly supplied by
Dr. E. Jansson, Helsinki) containing isolates from 2 RA cases (Jansson, 1971). A sucrose density gradient centrifugation (Williams, 1968) was performed using synovial fluid from one patient. Material from the visible band in the middle of the gradient was removed from the tube with a syringe and cultured in the same way as the synovial fluid from this patient.

\section{CULTURE MEDIA}

The media used was that described by Jansson (1971) with slight modifications. Brain-heart infusion broth was enriched with $20 \%$ pooled inactivated human serum, $2.5 \%$ yeast extract, $1 \%$ glucose, $20 \mu \mathrm{g} / \mathrm{ml}$ DNA, $0.5 \%$ pasteurized egg yolk, 500 units/ml penicillin, and HEPES buffer. The pH was adjusted to $7 \cdot 2$. For the solid medium $1.1 \%$ Ionagar was added to the above broth and the egg yolk was omitted.

\section{ISOLATION TECHNIQUE}

Broth cultures were made directly at the bedside where possible. Synovial fluid was aspirated with a sterile syringe from the patient's knee and $0.5 \mathrm{ml}$ inoculated into $5 \mathrm{ml}$ of enriched brain-heart infusion broth. After 7 to 10 days' incubation at $37^{\circ} \mathrm{C}$ a subculture was made into fresh broth again using a 1:10 dilution in an attempt to dilute out, with this early subculture, any inhibitory factors in the synovial fluid. After incubation for 6 weeks, 8 drops of the second broth culture were placed in the centre of a small agar plate and dried. Agar cultures were incubated in a perspex candle jar and examined after 3 to 7 days. Uninoculated broths with and without egg yolk were used as controls. Two blood agar plates were inoculated from each broth and incubated both aerobically and in an 
atmosphere of $8 \% \mathrm{CO}_{2}$. The incubation temperature was later lowered successfully to $35^{\circ} \mathrm{C}$.

\section{BIOCHEMICAL STUDIES}

(a) Arginine metabolism

Phenol red and $1 \%$ arginine were added to the enriched broth to test for arginine metabolism.

(b) Incorporation of ${ }^{32} \mathrm{PO}_{4}$ and gel electrophoresis of $\mathrm{RNA}$ extracts

$0.5 \mathrm{mCi}^{32} \mathrm{PO}_{4}$ (Amersham Radiochemical Centre) was added to broth cultures of Mycoplasma hominis, Mycoplasma fermentans, and to a culture from the synovial fluid of an RA patient. This latter culture had produced colonies on agar and the isolate was known as isolate RPL. After incubation at $35^{\circ} \mathrm{C}$ for 3 days the cultures were centrifuged and the sediment washed thoroughly with phosphate buffered saline (PBS). The washed sediment was resuspended in PBS and aliquots counted using liquid scintillant and a Beckman LS 100 counter. A monolayer culture of human fibroblasts growing in tissue culture 199 medium enriched with human serum was also labelled with ${ }^{32} \mathrm{PO}_{4}$ and aliquots of trypsinized cells counted. Uninoculated broth labelled with ${ }^{32} \mathrm{PO}_{4}$ was used as a control. Results were expressed as total counts incorporated in sedimented material per culture.

Some sediment from the above experiment was extracted for RNA and the major RNA fractions were visualized by gel electrophoresis.

\section{ELECTRON MICROSCOPY}

Two diluted joint fluids were prepared for electron microscopy using phosphotungstic acid to stain negatively the sedimented particles. These fluids had produced isolates on culture. Most of the cellular debris was first removed by low speed centrifugation.

The method used in preparation for scanning electron microscopy was developed by Dr. S. Rao (personal communication). Broth cultures were centrifuged at 5700 r.p.m. for 5 minutes, most of the supernatant fluid pipetted off, and the remaining 3 drops placed on a warmed prepared Formvar-coated stub, which was kept at $37^{\circ} \mathrm{C}$ for 1 hour. The sediment was then rinsed in buffered salt solution, and fixed with $2.5 \%$ glutaraldehyde in cacodylate buffer. After 30 minutes it was rinsed with buffer and dehydrated in ascending grades of acetone. After critical point drying the preparation was coated with carbon and finally with gold and was examined in a Siemans Autoscan.

The following material was prepared for scanning electron microscopy. (1) 3 broth cultures (from 3 different patients) incubated for 6 weeks which had produced colonies when inoculated on agar. (2) One culture which failed to produce colonies and was considered negative. (3) 3 control broths, 2 with and 1 without egg yolk. Each had been incubated in parallel with different series of cultures. (4) An actively growing culture of M. hominis isolated in this laboratory.

\section{Results}

Colonies were isolated from 9 synovial fluids from the 11 RA cases. Fluid from the patient with ankylosing spondylitis was negative. Fig. 1 shows the microscope appearance of colonies on agar. All isolates were similar except for a slight variation in size. Fig. 2 shows colonies grown from broth culture 56-M, which appear identical in size and morphology to our own isolates.

The small dense colonies usually appeared in groups, but with sparse overall growth. There was no increase in the number of colonies when an agar block was passaged to fresh agar, or when a colony was spread with a loop. Colonies varied in size from

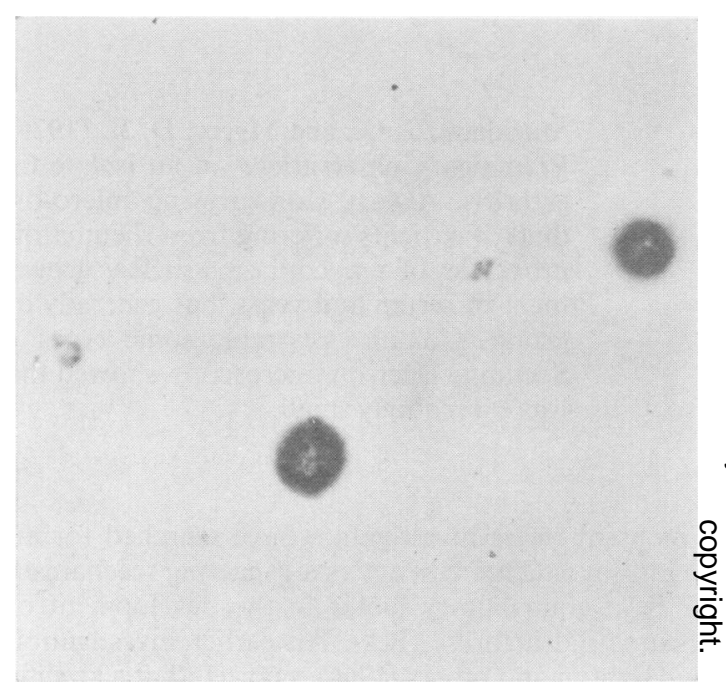

FIG. 1 Colonies of isolate from synovial fluid of an $R A$ patient. $\times 145$

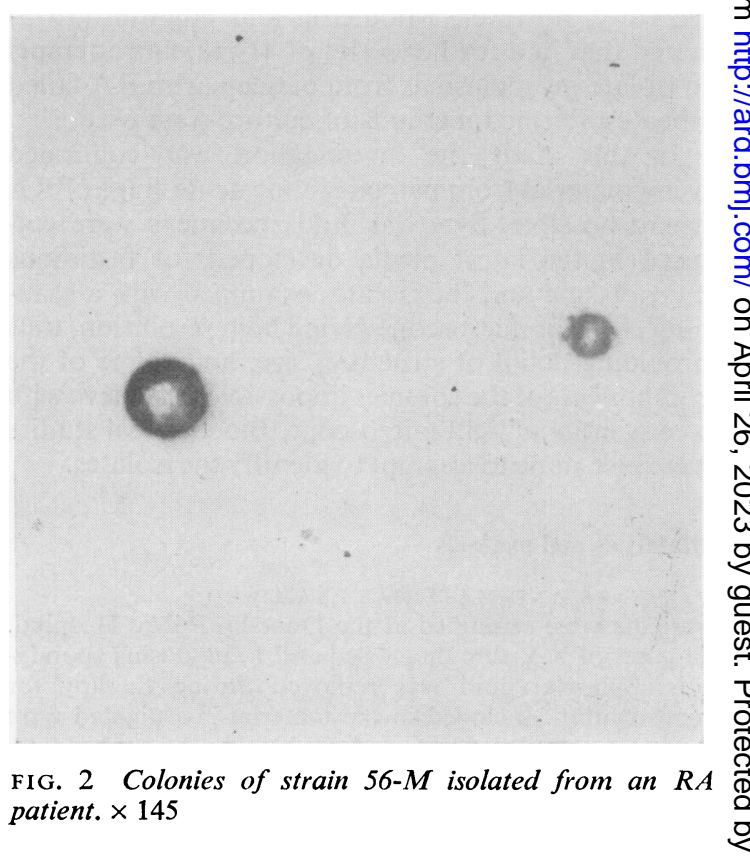


15-50 $\mu \mathrm{m}$, resembling some types of T-mycoplasma in size and morphology, but did not increase in size with longer incubation.

The broth culture inoculated with sucrose density gradient material and that from the synovial fluid of the same patient both produced colonies on agar although the former appeared earlier, possibly due to a higher concentration of organisms in the inoculum. There was no growth on blood agar plate cultures.

\section{ARGININE TEST}

Three strains were tested for arginine metabolism and each showed a colour change after 6 weeks' incubation.

INCORPORATION OF ${ }^{32} \mathrm{PO}_{4}$

The control broth gave residual counts of $<50$ counts/min (cpm), isolate RPL and $M$. fermentans $400 \mathrm{cpm}, M$. hominus $1850 \mathrm{cpm}$, and the fibroblasts $20000 \mathrm{cpm}$. The fibroblast counts represent the amount of incorporation for approximately $10^{6}$ cells, while those for the broth cultures represent an unknown number of organisms.

\section{GEL ELECTROPHORESIS OF RNA EXTRACTS}

Preliminary experiments using gel electrophoresis to separate the major forms of RNA from extracts of the sedimented material used in the ${ }^{32} \mathrm{PO}_{4}$ experiment indicated that isolate RPL, M. fermentans, and $M$. hominis gave similar patterns. The fibroblast RNA pattern was different.

\section{ELECTRON MICROSCOPY}

Of the two fluids examined by transmission electron microscope using the phosphotungstic acid prepar- ation, one fluid contained 'mycoplasma-like particles' as defined by size, membrane-bound appearance, and filamentous projections (Fig. 3), while the other fluid showed evidence only of 'cellular debris'.

The scanning electron microscope appearance of colonies of the isolate from 3 patients (RPL, LL, BM) is shown in Figs. 4-6. Fig. 7 shows the colony isolated from the broth culture 56-M. No colonies were found in material from the control broths and the negative culture by scanning. The latter was from the patient with ankylosing spondylitis. A colony of $M$. hominis is shown for comparison with one of an isolate at the same magnification (Figs, 8 and 9).

\section{Discussion}

Although agar cultures were inoculated from the second broth passage at $1,2,3,4,5$, and 6 weekly intervals, only the latter produced colonies. The incubation period for different species of mycoplasma varies greatly, and if this isolate is a mycoplasma it is reasonable to suppose that it is a very slow-growing strain.

The necessity for longer incubation periods for primary isolation of mycoplasmas was shown by Bradbury and Howell (1974) who found that a higher percentage of isolates resulted from incubation of primary cultures for up to 24 days. Since these colonies failed to increase in size in spite of continued incubation it could be concluded that they had become nonviable. It is possible that colonies were produced in the broth but when transferred to agar medium showed little increase in growth on the solid

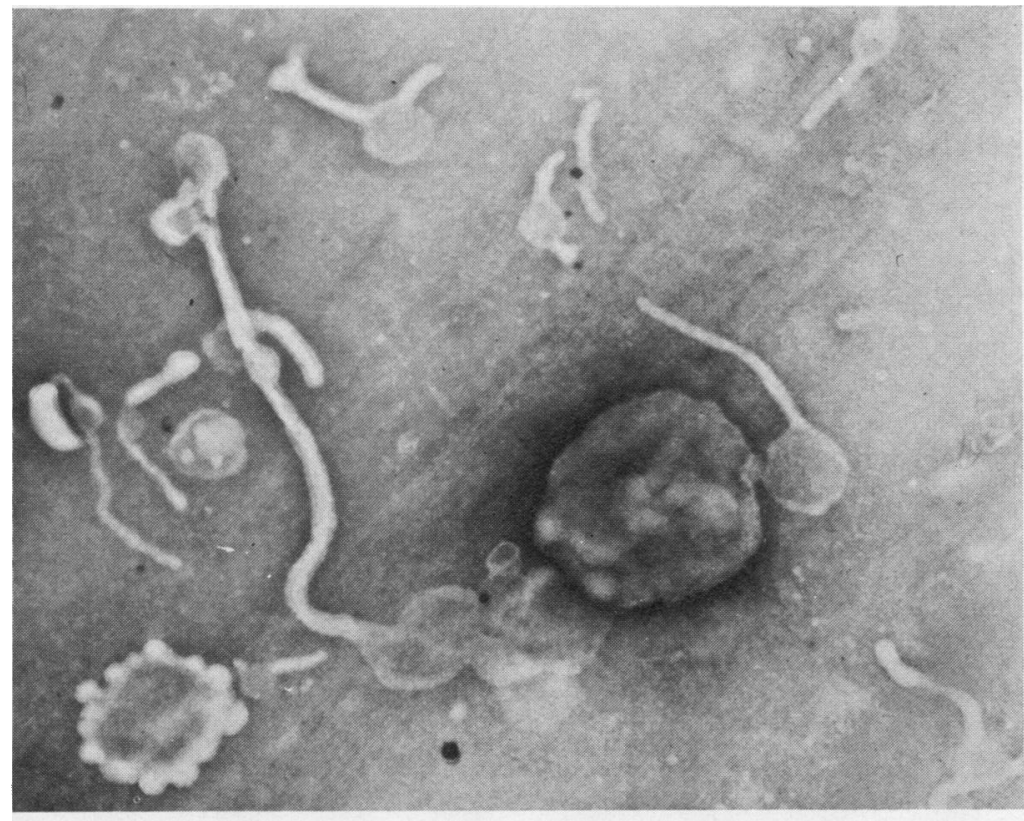

FIG. 3 Negatively-stained particles from an RA patient. $\times 32000$ 


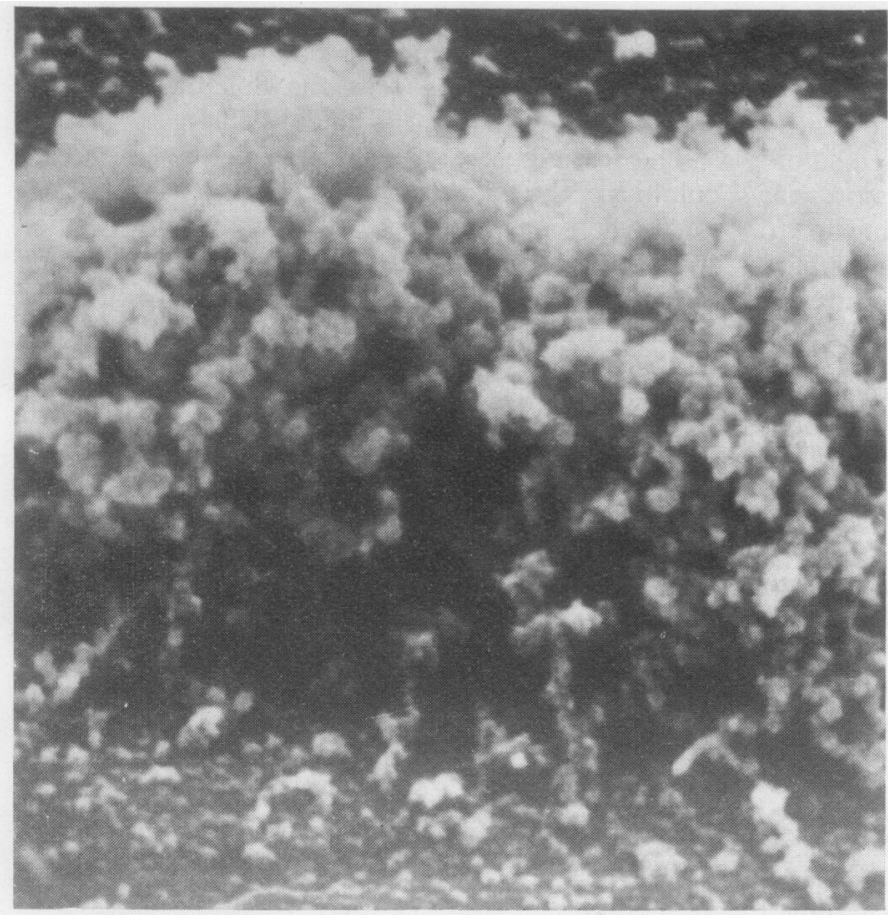

FIG. 4 Large colony of isolate RPL. SEM $\times 12400$

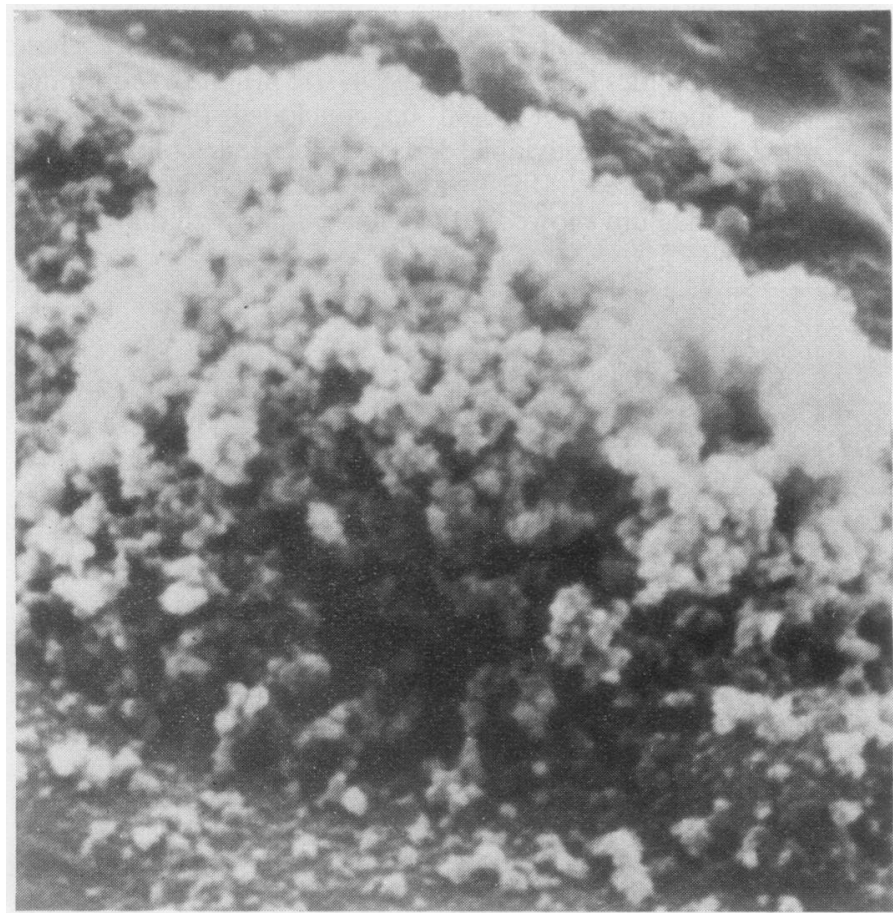

FIG. 5 Laige colony of isolate LL. SEM $\times 12400$

substrate. Successive broth subcultures appeared to remain viable, indicating that a liquid environment was essential for continuing growth. The results of the experiment using radioactive material indicated that incorporation of ${ }^{32} \mathrm{PO}_{4}$ was taking place in the broth cultures, but it was slow compared with that in a fibroblast culture.

In the scanning electron microscope photographs 


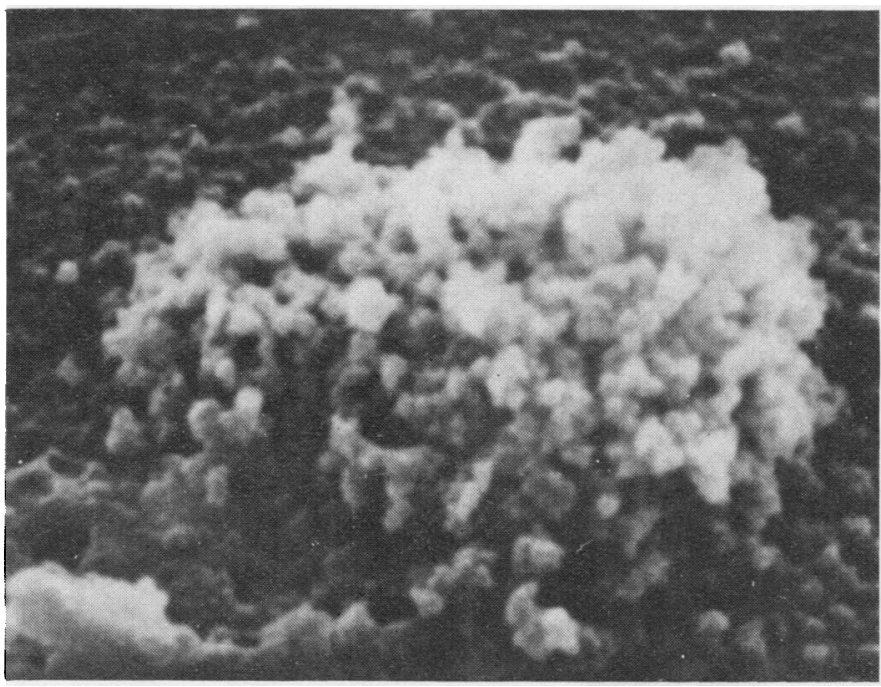

FIG. 6 Small colony of isolate BM. SEM $\times 12400$

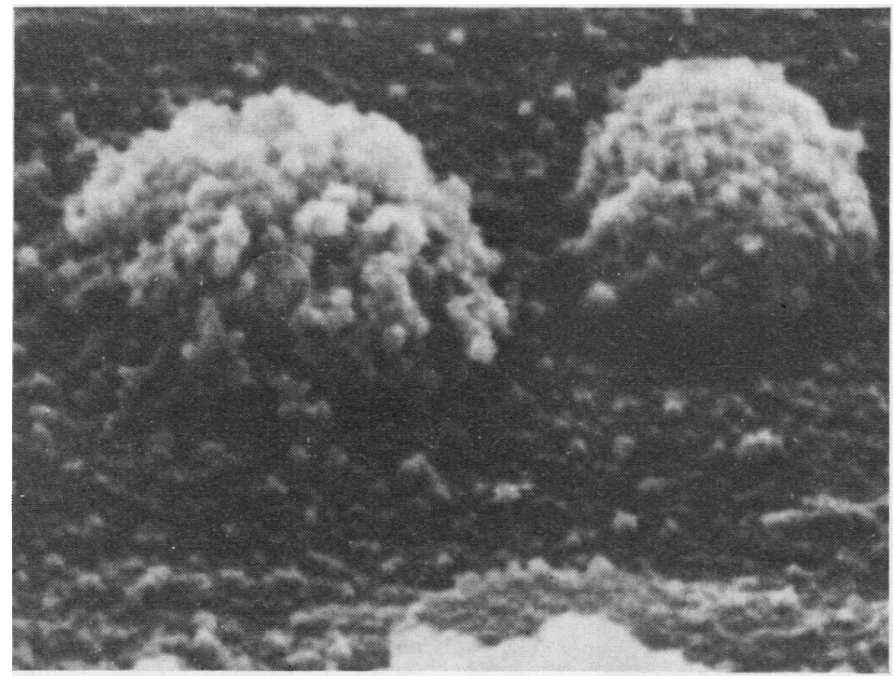

FIG. 72 small colonies of isolate 56-M. $\mathrm{SEM} \times 12400$

some components of colonies of the isolate were smaller, more irregular in shape, and more flattened and empty than those of a colony of $M$. hominis. The average diameter of a spherical mycoplasma cell is $0.5 \mu \mathrm{m}$ but viable units as small as about $0.3 \mu \mathrm{m}$ are found (Morowitz and Wallace, 1973). The size of the small components of colonies of these isolates was calculated at $0.2-0.4 \mu \mathrm{m}$, which might explain why colonies could not be propagated on solid medium. It is possible that the very small components are the 'elementary bodies' which many workers claimed as a method of reproduction. In 4-day-old cultures Jansson and others (1972) observed very small cells resembling elementary bodies, $0 \cdot 10-0 \cdot 15 \mu \mathrm{m}$ in size, and at 10 days they had formed raspberry-like clusters.

Klainer and Pollack (1973), in a scanning electron microscope study of many species of mycoplasma, found among aggregates of cells measuring approximately $1.0 \mu \mathrm{m}$ in diameter that there were clumps of unidentified smaller bodies $(0 \cdot 1 \mu \mathrm{m})$, but there was no evidence that these small bodies were viable.

Failure to propagate the colonies of this isolate may have been due to the lack of a necessary growth factor in the medium. Further work is being carried out on enrichment of the medium, and sectioning of organisms for examination by the electron microscope.

Bartholomew (1965) isolated 14 mycoplasma strains from 17 patients with several connective tissue diseases using tissue culture methods. With direct inoculation into PPLO medium his results were negative and he concluded that, 'if these mycoplasma strains did arise from the original specimen, one must 


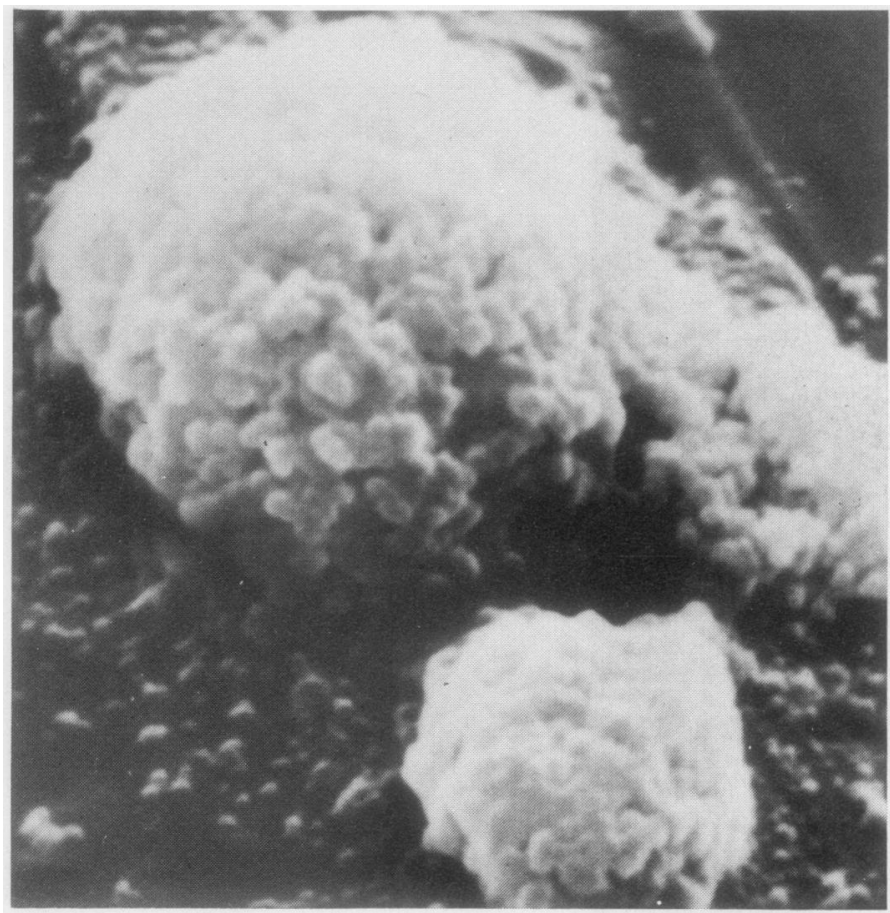

FIG. 8 Colony of M. hominis. $\times 8300$

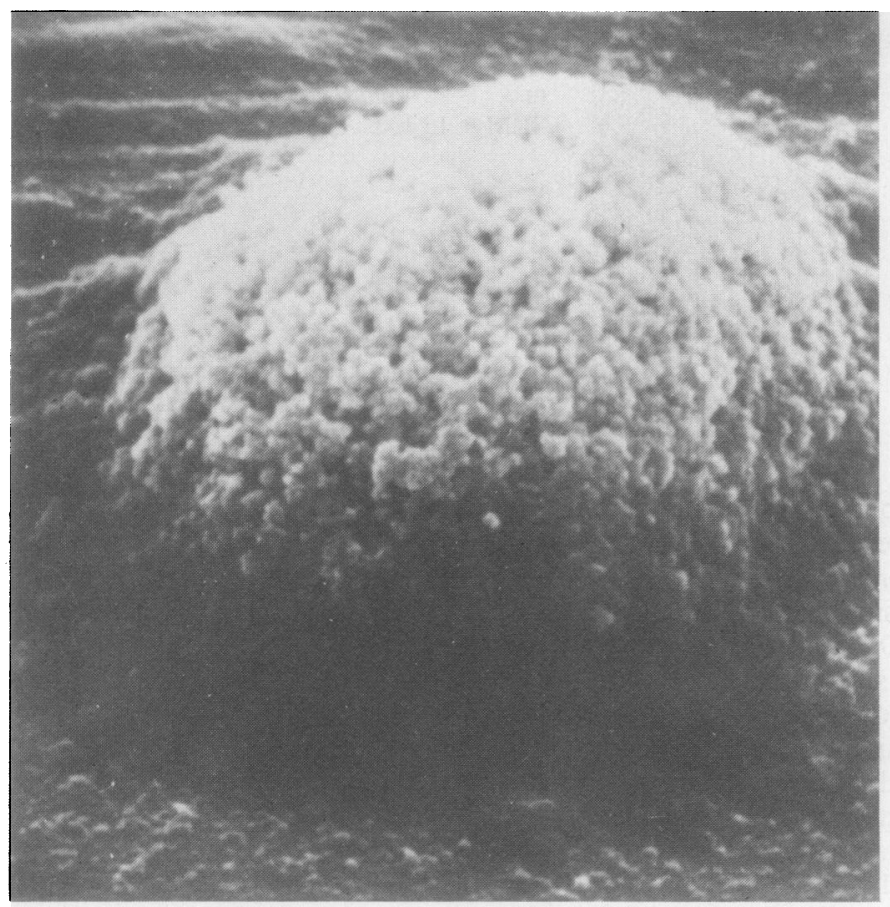

FIG. 9 Colony of isolate BM. Compared with $\mathrm{M}$. hominis the components are smaller and less rounded. $\times 8300$

assume thay are extremely fastidious, and they may require a period of adaption within or in proximity to living cells before they can grow in cell-free medium'.

The results of Fraser and others (1971) indicate that rheumatoid tissue is apt to carry very small numbers of mycoplasma organisms which must be practically nonantigenic. He suggests the possibility that poorly antigenic organisms, by persisting somewhere in the 
immune system, alter immunological function in some way other than by inducing a specific immune response.

In this investigation the absence of growth in control broths, the fact that the isolates are not strains of the common type of laboratory contaminant, and the recovery of the same colony type from several patients indicate that these are not artefacts.
We thank Associate Professor T. C. Highton for cooperation and helpful criticism; Dr. Elli Jansson (Helsinki) for continual encouragement and advice; Dr. S. Rao and his technical assistant Mr. B. Partridge for preparation and photography in the scanning electron microscopy work; and Mr. M. Bodger, Biochemistry Department, University of Otago, for assistance in the gel electrophoresis experiments. D. B. M. is supported by the New Zealand Medical Research Council.

\section{References}

BARTHOLOMEW, L. E. (1965) Arthr. and Rheum., 8, 376 (Isolation and characterization of mycoplasmas (PPLO) from patients with rheumatoid arthritis, systemic lupus erythematosus, and Reiter's syndrome)

BradbuRY, J. M., AND Howell, L. J. (1974) Res. vet. Sci., 17, 408 (Delayed growth and atypical colony formation of Mycoplasma synoviae)

Fraser, K. B., Shirodaria, P. V., Haire, M., and Middleton, D. (1971) J. Hyg., 69, 17 (Mycoplasmas in cell cultures from rheumatoid synovial membranes)

Highton, T. C., Caughey, D. E., Berkley, B. B., and Middleton, P. J. (1966) Ann. rheum. Dis., 25, 477 (Relationship between mycoplasma infection and rheumatoid arthritis and similar diseases)

JANsSON, E. (1971) J. clin. Path., 24, 53 (Isolation of fastidious mycoplasma from human sources)

-, Vainio, U., Snellman, O., AND TuUri, S. (1971) Ann. rheum. Dis., 30, 413 (Search for mycoplasma in rheumatoid arthritis)

-, Eklund, H., Vainio, U., Halme, H. ANd TuURI, S. (1972) Scand. J. Rheum., 1, 113 (Scanning electron microscopy of mycoplasma isolated from systemic lupus erythematosus disseminatus)

Klainer, A. S., and Pollack, J. D. (1973) Ann. N.Y. Acad. Sci., 225, 236 (Scanning electron microscopy techniques in the study of the surface structure of mycoplasmas)

Middleton, P. J., AND Highton, T. C. (1975) Unpublished (Failure to demonstrate mycoplasmas and cytopathogenic virus in rheumatoid arthritis)

Morowitz, H. J., AND Wallace, D. C. (1973) Ann. N.Y. Acad. Sci., 225, 62 (Genome size and life cycle of the mycoplasma)

Williams, M. H. (1968) 'Recovery of mycoplasma from rheumatoid synovial fluid', in 'Rheumatic Diseases', ed. J. J. R. Duthie and W. R. M. Alexander, p. 172. Pfizer Monograph No. 3. University Press, Edinburgh 